Bulgarien,

Dänamark,

China,

Deutschland,

Finnland,

Großbritannien,

Iran,

Italien,

Japan,

Kroatien,
Polen,

Rumänien,

Rußland,

Schweiz,

Slowakei,

Slovenien,

Tschechische Republik,

Türkei,

Ungarn,

USA

haben diese Veranstaltung besucht. An jedem Tag wurden etwa 30 Vorträge abgehalten. Für Diskussionen stand jeweils ausreichend Zeit beim mittäglichen Arbeits-Imbiß und beim Heurigenabend sowie beim Empfang im Wr. Rathaus zur Verfügung.

Metrology for Quality Control in Production: Die Meßtechnik ist eine „Enabling Science“ - eine Wissenschaft, welche die Basis für präzise Fertigung und weitere Entwicklungen bildet.

Viele Milliarden Dollar werden jährlich in die Entwicklung

der Meßtechnik investiert. Die Herstellung von HiTech-Geräten (z. B. Mobiltelefone, Air-Bags oder Videorecorder) ist ohne die Anwendung modernster und genauester Meßmethoden nicht denkbar.

Die Entwicklung von Qualitätsmanagementsystemen erfordert in hohem Maße die Einbindung der Meßtechnik in die Fertigung. Die Anwendung der Meßtechnik entwickelt sich hierbei von Kontrollmessungen am Ende des Fertigungsprozesses weg in Richtung prozeßbegleitender und prozeßintegrierter Meßtechnik. So kann man etwa seit 1970 die vermehrte Einbeziehung von computerunterstützten Meßtechnologien (z. B. Koordinatenmeßtechnik) beobachten. Ein Ende der Entwicklung der MeBtechnik - sei es im Hinblick auf die Genauigkeit oder im Hinblick auf ihre Bedeutung ist nicht absehbar.

In der April-Ausgabe 1999 der e \& i wird noch genauer über diese Veranstaltung berichtet werden; insbesondere ist vorgesehen, ausgewählte Beiträge zu einigen speziellen Themen in vollständiger Form zum Abdruck zu bringen.

\title{
Zentralstatistik elektrischer Unfälle für das Jahr 1997
}

Mitgeteilt vom Bundesministerium für wirtschaftliche Angelegenheiten (in gekürzter Form wiedergegeben)

\section{Unfälle durch Elektrizität}

1.1 Gesamtanzahl der erfaßten Unfälle durch elektrischen Strom

1997

$$
\begin{aligned}
& \text { Anzahl der } \\
& \text { Unfälle } \\
& \text { gesamt }
\end{aligned}
$$

172
Mit tödlichem

Ausgang

14

\subsection{Art der Unfälle}

Arbeitsunfall .................. 150

Wegunfall $\ldots \ldots \ldots \ldots \ldots \ldots \ldots \ldots \ldots \ldots \ldots$.

Privatunfall ... . . . . . . . . . . . . . . . . . . 19

Absichtlich herbeigeführter Unfall ..... 1

Unbekannt $\ldots \ldots \ldots \ldots \ldots \ldots \ldots \ldots \ldots .2$

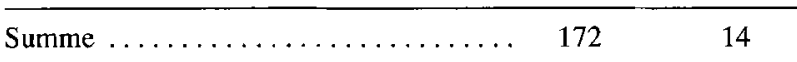

\begin{tabular}{|c|c|c|}
\hline $\begin{array}{l}\text { Männlich } \ldots \ldots \ldots \ldots \ldots \ldots \ldots \ldots \ldots \ldots \ldots \ldots \ldots \ldots \\
\text { Weiblich } \ldots \ldots \ldots \ldots \ldots \ldots \ldots\end{array}$ & $\begin{array}{r}156 \\
16\end{array}$ & 14 \\
\hline Summe & 172 & 14 \\
\hline
\end{tabular}

\subsection{Geschlecht der Verletzten}

\subsection{Berufsarten}

Elektroingenieure und Betriebsleiter . . . . 3

Elektromeister $\ldots \ldots \ldots \ldots \ldots \ldots \ldots \ldots . \ldots \ldots, 12$

Obermonteure .................. 2

Monteure (auch Elektroinstallateure,

Elektriker) . . . . . . . . . . . .

Hilfsmonteure (Angelernte)

Lehrlinge

-

1

14

(




$\begin{array}{cc}\text { Anzahl der } & \text { Mit } \\ \text { Unfälle } & \text { tödlichem } \\ \text { gesamt } & \text { Ausgang }\end{array}$

Spannungführende Teile mit nicht spannungführenden verwechselt

Körperschluß Masseschluß .........

Mangelhafte Isolation, Leitung...$\ldots \ldots$

Sonstige Mängel der Anlage

(Lichtbogen ...) ...............

Unvorschriftsmäßige Geräte (Pfusch) ....

Mangelhafte Werkzeuge

(ohne Gummihandschuh)

Kurzschluß (erzeugt mit Werkzeug,

Schrauben)

Schrittspannung $\ldots \ldots \ldots \ldots \ldots \ldots \ldots$

Brandbekämpfung, Löscharbeiten ..... .

Offene Lampenfassungssicherung $\ldots \ldots$.

Überschläge (nicht auf den Verletzten) ...

Leitungsrisse, herabhängende Drähte ... .

Gedankenlosigkeit, Unvorsichtigkeit .....

Weidezäune $\ldots \ldots \ldots \ldots \ldots \ldots \ldots$.

Elektrofischen.$\ldots \ldots \ldots \ldots \ldots \ldots \ldots$

Sonstige $\ldots \ldots \ldots \ldots \ldots \ldots \ldots \ldots$

Unbekannt $\ldots \ldots \ldots \ldots \ldots \ldots \ldots \ldots$

Summe $\ldots \ldots \ldots \ldots \ldots \ldots \ldots \ldots$

$\begin{array}{rr}2 & - \\ 1 & - \\ 13 & 3 \\ 6 & - \\ 3 & - \\ 1 & - \\ - & - \\ 1 & - \\ - & - \\ - & - \\ 2 & - \\ - & - \\ - & - \\ 104 & 8 \\ - & - \\ \overline{2} & - \\ 30 & - \\ 172 & 14\end{array}$

\subsection{Stromart}

Gleichstrom $\ldots \ldots \ldots \ldots \ldots \ldots \ldots \ldots$

Wechselstrom .................

Drehstrom .................... 61

Blitz .......................

Sonst. statische Elektrizität, Induktion .....

Unbekannt $\ldots \ldots \ldots \ldots \ldots \ldots \ldots \ldots$

\begin{tabular}{llr}
\hline Summe $\ldots \ldots \ldots \ldots \ldots \ldots \ldots \ldots \ldots \ldots$ & 172 & 14 \\
\hline
\end{tabular}

\subsection{Höhe der Spannung}

\begin{tabular}{|c|c|c|}
\hline Hochspannung über $100 \mathrm{kV}$ (höchst). & 3 & 1 \\
\hline Hochspannung von $1 \mathrm{kV}$ bis $100 \mathrm{kV} \ldots \ldots$ & 33 & 5 \\
\hline Mittelspannung von $250 \mathrm{~V}$ bis $1 \mathrm{kV}$ & 58 & 1 \\
\hline $\begin{array}{l}\text { Niederspannung von } 42 \mathrm{~V} \text { bis } 250 \mathrm{~V} \ldots \ldots \\
\text { Niederspannung bis } 42 \mathrm{~V} \text { (Klein- }\end{array}$ & 64 & 5 \\
\hline $\begin{array}{l}\text { spannung) } \ldots \ldots \ldots \ldots \ldots \\
\text { Kleinspannung bis } 24 \mathrm{~V} \text { (Kleinst- }\end{array}$ & - & - \\
\hline spannung) $\ldots \ldots \ldots \ldots \ldots \ldots \ldots$ & 3 & - \\
\hline 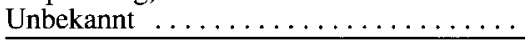 & 11 & 2 \\
\hline Summe & 172 & 14 \\
\hline
\end{tabular}

\subsection{Stromdurchgang}

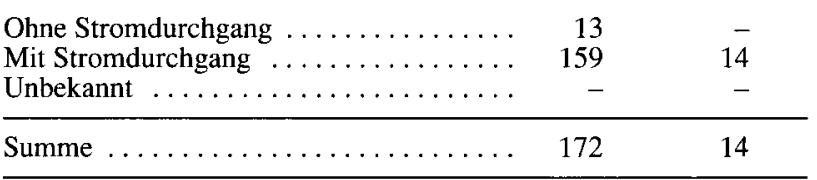

\subsection{Art der Verletzung}

Keine Verletzung $\ldots \ldots \ldots \ldots \ldots \ldots \ldots$

Strommarke .........................

Verbrennung .................. 59

Blendung $\ldots \ldots \ldots \ldots \ldots \ldots \ldots \ldots$

Schock ....................... 19

Mechanische Verletzung ............ 6

Metallisation ................. 11

Mehrere Verletzungsarten $\ldots \ldots \ldots \ldots \ldots, 25$

Sonstige Verletzungen ...............

Unbekannt ................. 12

Summe $\ldots \ldots \ldots \ldots \ldots \ldots \ldots \ldots, 172$

\section{Blitzunfälle}

\subsection{Gesamtanzahl der Blitzunfälle}

1997

\subsection{Berufsarten}

Land- und Forstwirtschaft $\ldots \ldots \ldots \ldots \ldots \quad 2 \quad 1$

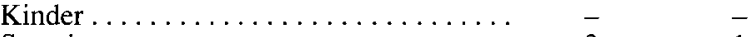

\begin{tabular}{|c|}
\hline Sonstige \\
\hline
\end{tabular}

Summe $\ldots \ldots \ldots \ldots \ldots \ldots \ldots \ldots \ldots 5_{2}$

\subsection{Unfallstelle}

Im Freien unter Bäumen u. dgl. . . ..... 32

Im Freien ohne besondere Anziehungs-

objekte $\ldots \ldots \ldots \ldots \ldots \ldots \ldots \ldots \ldots, \quad 1 \quad-$

\begin{tabular}{lll} 
In Gebäuden $\ldots \ldots \ldots \ldots \ldots \ldots \ldots \ldots \ldots \ldots$ & 1 & - \\
\hline Summe $\ldots \ldots \ldots \ldots \ldots \ldots \ldots$ & 5 & 5
\end{tabular}

\subsection{Stromdurchgang}

Ohne Stromdurchgang $\ldots \ldots \ldots \ldots \ldots \ldots \overline{5} \overline{5}$

Unbekannt $\ldots \ldots \ldots \ldots \ldots \ldots \ldots \ldots \ldots \ldots-$

Summe $\ldots \ldots \ldots \ldots \ldots \ldots \ldots \ldots, 5 \ldots \ldots \ldots$

\subsection{Art der Verletzung}

Keine Verletzung $\ldots \ldots \ldots \ldots \ldots \ldots \ldots \ldots-$

Strommarke $\ldots \ldots \ldots \ldots \ldots \ldots \ldots \ldots \ldots z_{-}^{-}-$

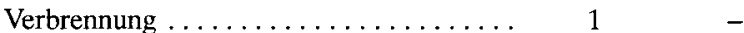

Blendung ...

Schock

Mechanische Verlet

Verletzung $\ldots \ldots \ldots \ldots$

Metallisation . . . . . . . . . . . . .

Mehrere Verletzungsarten $\ldots \ldots \ldots \ldots \ldots$

\begin{tabular}{l} 
Mehrere Verletzungsarten $\ldots \ldots \ldots \ldots \ldots$ \\
Sonstige Verletzungen $\ldots \ldots \ldots \ldots \ldots \ldots \ldots$ \\
Unbekannt $\ldots \ldots \ldots \ldots \ldots \ldots \ldots \ldots \ldots \ldots$ \\
\hline
\end{tabular}

Unbekannt $\ldots \ldots \ldots \ldots \ldots \ldots \ldots \ldots \ldots, \overline{1}$

Summe $\ldots \ldots \ldots \ldots \ldots \ldots \ldots \ldots \ldots \ldots \ldots \quad 5 \quad 2$ 